\title{
Comparison of the efficacy and safety of antiviral agents for COVID-19: a systematic review and meta-analysis
}

\author{
Hongwei Peng ${ }^{1}$, Lili Liu ${ }^{1}$, Zhangren Chen ${ }^{1}$, Jinhua Wen ${ }^{1}$, Jiaming Liu ${ }^{2}$, Mengiun Zhao ${ }^{3}$, Feifan Ouyang ${ }^{3}$, Xinyu X ${ }^{3}$, \\ Tiantian $\mathrm{Xu}^{1}$, Li Cao ${ }^{1, *}$, Xiaohua Wei ${ }^{1}$ and Tao Hong ${ }^{4, *}$ \\ ${ }^{1}$ Department of Pharmacy, the First Affiliated Hospital of Nanchang University, P.R. China \\ 2 Department of Orthopedics, the First Affiliated Hospital of Nanchang University, P.R. China \\ ${ }^{3}$ The First Clinical Medical College of Nanchang University, P.R. China \\ ${ }^{4}$ Department of Neurosurgery, the First Affiliated Hospital of Nanchang University, P.R. China \\ ${ }^{*}$ Correspondence: caoli_ndyfy@sina.com (Li Cao) \\ ht2000@vip.sina.com (Tao Hong)
}

\section{DOI: $10.31083 /$ i.jmcm.2020.01.003}

This is an open access article under the CC BY 4.0 license (https://creativecommons.org/licenses/by/4.0/).

Objective: To compare the efficacy and safety of antiviral agents currently studied for the treatment of the COVID-19 pandemic. Methods: A literature search was conducted on the PubMed, EMBASE, Web of Science, CNKI (Chinese Database), and MedRxiv for studies published from 1966 till May 10, 2020, and identified articles containing "COVID-19" and "antiviral agents". Studies were reviewed and screened in the guidance of PRISMA. STATA 15.1 software was used to build a random-effects model. Heterogeneity was assessed using $1^{2}$. The Cochrane Risk of Bias or Newcastle-Ottawa-Scale (NOS) was employed to evaluate the public bias. Results: We identified 916 papers and included 7 studies involving 878 patients. The network meta-analysis was centered on comparing the efficacy and safety of presently used antiviral drugs for COVID-19. Among the antiviral agents applied in the treatment of COVID-19 treatment, including lopinavir/ritonavir, remdesivir, favipiravir, arbidol (umefenovir) or placebo, favipiravir exhibited significantly better efficacy in nucleic acid conversion rate [RR 2.38, $95 \% \mathrm{Cl}(1.05,5.41)]$ and $\mathrm{CT}$ improvements [RR 1.85, $95 \% \mathrm{Cl}(1.07,3.2)]$ than arbidol as well as other included antivirals though no significant association were found. ARB had advantages in nucleic acid conversion rate and ADRs incidence. Besides, favipiravir was more superior in safety than other antiviral drugs assessed by SUCRA (Surface Under the Cumulative Ranking Curve) ranking. Conclusions: Favipiravir had better efficacy in clinical recovery as well as more acceptable safety, though further clinical research should be designed to confirm these results. With the limited data of remdesivir, we could not conclude a statistical advantages of using remdesivir.

\section{Keywords}

COVID-19; antiviral agents; efficacy; safety; network meta-analysis

\section{Introduction}

At the end of 2019, a new type of pneumonia emerged in Wuhan, central China, and rapidly spread through the entire world. Lacking effective drugs for this disease significantly hindered the treatment at the very beginning. Not until Lu Roujian et al. unveiled the results of pathogen isolation and genomic characterization [1], a new human-infected beta-coronavirus was found and named as SARS-CoV-2 by ICTV (International Committee on Taxonomy of Viruses, ICTV) [2]. Currently, coronavirus infectious disease-19 (COVID-19) has been announced as a pandemic by the WHO and became the focus of the world as the number of patients infected with COVID-19 has increased up to over 4,400,000, with a mortality rate over $6 \%$ by May 15th, 2020. SARS-CoV-2 shares some similarities with MERS-CoV (with about 50\% identity) and SARS-CoV (about 79\% identity), and notably it shares a similar receptor-binding domain structure to that of SARS-CoV. Consequently, antiviral drugs that had proved to be effective in the previous SARS or MERS treatment have been tested in COVID-19 treatment, though SARS-CoV and MERS$\mathrm{CoV}$ disappeared suddenly and no further antiviral studies were continued. To deal with the pandemic crisis, scientists have registered hundreds of clinical trials around world, with varied antiviral drugs, study designs, and endpoints et al.

Additionally, preliminary results showed that remdesivir (REM) had vigorous antiviral activity against SARS-CoV-2 in vitro [3]. Besides, due to lack of effective agents, some other antiviral agents, such as darunavir (DRV), was used to test the efficacy against SARS-CoV-2. However, in vitro study showed DRV had no antiviral activity against SARS-CoV-2 at clinically relevant concentrations (EC50 > 100 $\mu \mathrm{M}$ ) [4]. Currently, the NIH COVID-19 treatment guidelines updated the antiviral section [5]: REM was recommended as the investigational antiviral agent as it has not yet been approved by the FDA and it was available through an FDA emergency use authorization for the treatment of COVID19 in hospitalized patients with severe disease. Some latest results of clinical trials have been released, and favipiravir (FPV) 
and arbidol (ARB; umefenovir) showed potential antiviral efficacy against COVID-19. However, due to the varied clinical research design, it is crucial to conduct a systematic review of the published data, in order to provide rationale evidence for clinicians in choosing anti-viral drugs to combate COVID-19.

\section{Materials and methods}

\subsection{Search strategy and selection criteria}

We searched MEDLINE, Embase, preprint database and the Cochrane Central Library for clinical trials and cohort studies published in English from 1966 till May 10, 2020. We also searched ClinicalTrials.gov and the chictr.org.cn of Controlled Trials to identify any clinical study that was not yet published, but potentially eligible for inclusion. Additionally, we also searched medRxiv, ChinaXiv.org for any clinical literature that meets our inclusion criteria.

\subsection{Study selection and data extraction}

We included studies of outcomes in the treatment of adults diagnosed with COVID-19. The diagnostic criteria were in accordance with Chinese clinical guidance for COVID-19 [6]. The treatment of the patients with antiviral agents, included Lopinavir/Ritonavir (LPV/RTV), arbidol (ARB; umefenovir), favipiravir (FPV), remdesivir (REM) or placebo $(\mathrm{CON})$, with at least two-arm design comparison. The outcome indicator included: (i) SARS-CoV-2 nucleic acid positive-to-negative conversion rate 7 days after initiating antiviral treatment. One of the vital diagnosis criteria included SARS-CoV-2 nucleic acid positivity by real-time reverse-transcriptase polymerase-chain-reaction (RT-PCR) assay for nasal and/or pharyngeal swab specimens. (ii) SARS-CoV-2 nucleic acid positive-to-negative conversion rate 14 days after initiating antiviral treatment. (iii) 7 days computerized tomography (CT) improvements: the efficacy of antiviral agents was assessed by chest CT 7 days after initiating antivirals treatment; (iv) 14 days CT improvements: chest CT assessment 14 days after initiating antivirals treatment; (v) Alleviation of cough: cough relief 7 days after antiviral treatment; (vi) Incidence of adverse effects: any abnormality examined by clinical laboratory testing or daily vital signs in the follow-up period; All the clinical outcomes were important indicators of clinical recovery listed in the Chinese Clinical Guidance for COVID-19 [6, 7]; (vii) Allcause mortality at day 7,14 or 28 after antiviral treatment initiating.

Two investigators (Peng Hongwei and Liu Lili) worked independently, searched and read all titles and abstracts and titles in duplicate to identify papers relevant to this study. When discrepancies occurred, a third coauthor (Chen Zhangren) made the final judgement. Peng and Liu assessed eligibility from full-text articles, with a similar process for potential disagreements as described above. Data extraction from included studies (i.e., published abstracts and manuscripts) was duplicated with a standardized extraction form. The characteristics of the research (study design, antiviral regimen, therapy duration, etc.), the outcomes of interest mentioned above, and biased risk assessment were extracted.

\subsection{Quality assessment}

We assessed the quality of individual clinical research using the Cochrane Risk of Bias instrument evaluating seven key domains [8]. We employed the Grading of Recommendations Assessment, Development and Evaluation (GRADE) system for rating over- all quality of evidence, using the four-step approach proposed to assess the quality of treatment effect estimates from network meta-analyses. For cohort study or case-control study, NewcastleOttawa-Scale (NOS) was employed [9]. We determined the quality of evidence for each primary outcome after considering each of these elements, and classified them as high, moderate, low, or very low quality [10].

\subsection{Statistical analyses}

We completed network meta-analysis using Stata 15.1 software. Heterogeneity was assessed by the $\chi^{2}$ test. In the cases of heterogeneity, sensitivity analysis was made and the heterogeneity sources were investigated. We analyzed two outcomes (CT improvements and nucleic acid conversion rate) at two timepoints: 7 days and 14 days after initiating treatment. Estimates of comparative efficacy and safety are represented as relative ratios (RR) with associated $95 \%$ confidence intervals (CI) for binary outcomes. We made a comparison of each intervention by surface under the cumulative ranking curve (SUCRA).

\section{Results}

\subsection{Literature search and results}

We have identified 916 publications through database searching and 2 additional records. Through titles and abstract reading, we finally included 7 articles for network meta-analysis. These articles included four randomized clinical trials and three cohort studies. The results of the literature search are depicted in Fig. 1. The evaluation of research bias was showed in Supplementary Fig. 1.

\subsection{Characteristics of the included literature and hetero- geneity test}

The characteristics of the research included are shown in Table 1 . The network relationships of the outcome indicators were shown in Fig. 2. We have analyzed 6 outcome indicators mentioned above and the heterogeneity test revealed no significant differences, so we therefore chose a random-effect model for further analysis.

\subsection{Network relationship analysis}

There were 7 studies reported the SARS-CoV-2 nucleic acid conversion rate.

\subsection{Network meta-analysis results}

3.4.1 Nucleic acid positive-to-negative conversion rate

There were 5 papers reporting the COVID-19 nucleic acid positive-to-negative conversion rates after different interventions. As shown in Fig. 2A, there were 4 direct comparison concerning LPV/RTV (LPV/RTV vs. FPV, LPV/RTV vs. LPV/RTV+ARB, LPV/RTV vs. CON, LPV/RTV vs. ARB), two studies compared the efficacy of LPV/RTV vs. ARB. The three interventions, LPV/RTV, CON, ARB formed a closed loop, indicating direct and indirect comparison could be made among these three interventions.

Fix effect network analysis showed that the nucleic acid conversion rate 7 days after initiating treatment in the LPV/RTV+ARB arm [RR 2.31, 95\% CI $(1.05,4.29)]$ was better than LPV/RTV monotherapy. Besides, the LPV/RTV treatment was inferior to the FPV [RR $0.25,95 \% \mathrm{CI}(0.13,0.48)]$ or ARB monotherapy [RR $0.59,95 \%$ CI $(0.36,0.96)]$. Additionally, FPV displayed better activity than ARB [RR 2.38, 95\%CI $(1.05,5.41)]$. There was 


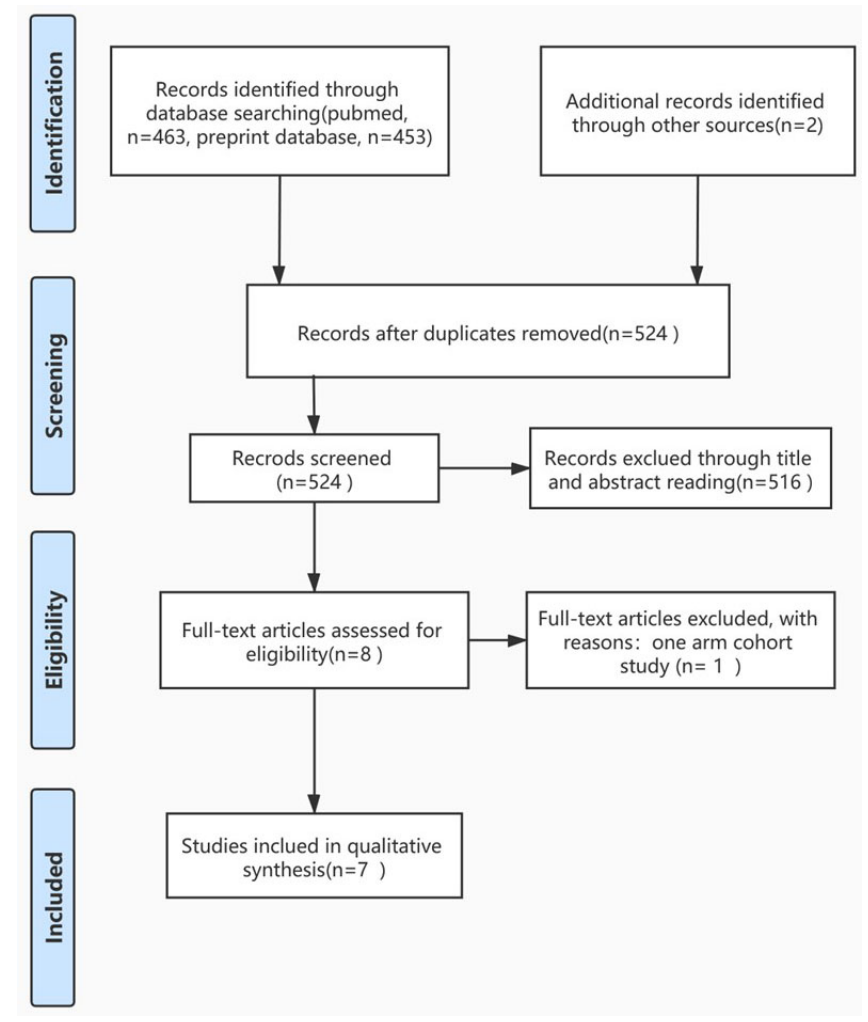

Figure 1. The PRISMA flow of literature searching and screening.

A

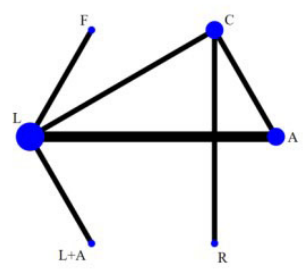

B

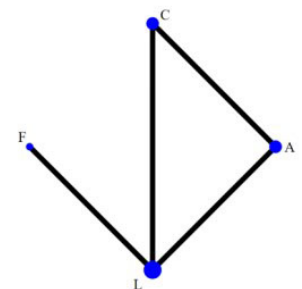

C

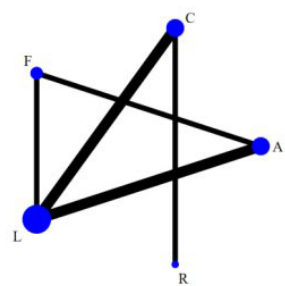

Figure 2. Network relationships of different outcomes. A. positive-to-negative conversion of SARS-CoV-2 nucleic acid; B. CT improvements; C. incidence of $A D R s$. $A=$ Arbidol; $C=$ Placebo or basic therapy; $F=$ Favipiravir; $L=$ Lopinavir/Ritonavir; $L+A=$ Lopinavir $/$ Ritonavir + Arbidol.

Table 1. Characteristics of included studies

\begin{tabular}{|c|c|c|c|c|c|c|}
\hline Author & Year & Design & Country & Antiviral therapy & $\begin{array}{l}\text { Number of } \\
\text { participants }\end{array}$ & Outcomes \\
\hline Zhu Zhen [11] & 2020 & cohort study & China & LPV/RTV versus ARB & $34 / 16$ & (i)(ii)(vi) \\
\hline Deng Lisi [12] & 2020 & retrospective cohort study & China & ARB plus LPV/RTV versus LPV/RTV & $16 / 17$ & (i)(ii)(iii) \\
\hline Li Yueping [13] & 2020 & Randomized, controlled study & China & LPV/RTV versus ARB versus basic therapy & $21 / 16 / 7$ & (i)(ii)(iii) (iv)(v)(vi) \\
\hline Chen Chang [14] & 2020 & randomized trial & China & FPV versus ARB & $116 / 120$ & $(\mathrm{v})(\mathrm{vi})$ \\
\hline Cai Qingxian [15] & 2020 & open-label control study & China & FPV versus LPV/RTV & $35 / 45$ & (i)(ii)(iii) (iv)(vi) \\
\hline Wang Yeming [16] & 2020 & $\begin{array}{l}\text { Randomized, double-blind, } \\
\text { placebo-controlled, multicenter }\end{array}$ & China & REV versus placebo & $158 / 78$ & (i)(ii) (vi)(vii) \\
\hline Cao Bin [17] & 2020 & $\begin{array}{l}\text { Randomized, double-blind, } \\
\text { placebo-controlled, multicenter }\end{array}$ & China & LPV/RTV versus basic therapy & $99 / 100$ & (i) (iii) (vi)(vii) \\
\hline
\end{tabular}

no significant difference when comparing between other interventions (Fig. 3A). When it comes to SUCRA ranking (7 days nucleic acid positive-to-negative conversion rate), the FPV arm obtained the highest SUCRA score, followed by LPV/RTV+ARB, REM, CON, ARB and LPV/RTV. In the outcome of 14 days' nucleic acid conversion rate, no statistical difference was found and the rank- 
A

\begin{tabular}{|l|l|l|l|l|l|}
\hline \multicolumn{1}{|c|}{ REM } & $0.56(0.19,1.67)$ & $0.99(0.42,2.33)$ & $0.51(0.18,1.47)$ & $0.94(0.56,1.59)$ & $0.7(0.3,1.64)$ \\
\hline $0.88(0.33,2.38)$ & LPV/RTV+ARB & $1.77(0.9,3.48)$ & $0.92(0.37,2.28)$ & $1.69(0.65,4.43)$ & $1.25(0.57,2.76)$ \\
\hline $1.87(0.93,3.78)$ & $\mathbf{2 . 1 3}(\mathbf{1 . 0 5 , 4 . 2 9 )}$ & \multicolumn{1}{c}{ LPV/RTV } & $0.52(0.28,0.95)$ & $0.95(0.48,1.9)$ & $0.71(0.47,1.06)$ \\
\hline $0.47(0.18,1.22)$ & $0.53(0.2,1.39)$ & $\mathbf{0 . 2 5}(\mathbf{0 . 1 3}, \mathbf{0 . 4 8})$ & \multicolumn{1}{|c|}{$\mathbf{F P V}$} & $1.77(0.9,3.48)$ & $1.36(0.66,2.83)$ \\
\hline $1.02(0.76,1.38)$ & $1.16(0.45,3)$ & $0.55(0.29,1.03)$ & $2.2(0.88,5.5)$ & \multicolumn{1}{c}{ CON } & $0.74(0.38,1.46)$ \\
\hline $1.11(0.57,2.14)$ & $1.26(0.54,2.96)$ & $\mathbf{0 . 5 9 ( 0 . 3 6 , 0 . 9 6 )}$ & $\mathbf{2 . 3 8 ( 1 . 0 5 , 5 . 4 1 )}$ & $1.08(0.6,1.94)$ & ARB \\
\hline
\end{tabular}

7 days nucleic acid conversion rate

14 days nucleic acid conversion rate

B

\begin{tabular}{|l|c|c|c|c|}
\hline LPV/RTV+ARB & NA & NA & NA & NA \\
\hline $2.34(1.04,5.26)$ & LPV/RTV & $\mathbf{0 . 6 8 ( 0 . 5 3 , 0 . 8 7 )}$ & $0.89(0.66,1.19)$ & $1.26(0.78,2.03)$ \\
\hline $1.62(0.62,4.2)$ & $0.69(0.41,1.16)$ & FPV & $1.31(0.89,1.92)$ & $\mathbf{1 . 8 5 ( 1 . 0 7 , 3 . 2 )}$ \\
\hline $1.32(0.5,3.46)$ & $\mathbf{0 . 5 7 ( 0 . 3 5 , 0 . 9 3 )}$ & $0.82(0.39,1.71)$ & CON & $1.41(0.89,2.24)$ \\
\hline $2.62(0.76,9.06)$ & $1.12(0.46,2.72)$ & $1.62(0.55,4.77)$ & $1.98(0.98,4)$ & ARB \\
\hline
\end{tabular}

C

7 days $\mathrm{CT}$ improvements

14 days $\mathrm{CT}$ improvements

\begin{tabular}{|l|c|c|c|c|}
\hline \multicolumn{1}{|c|}{ REM } & NA & NA & NA & NA \\
\hline $0.72(0.02,26.09)$ & LPV/RTV & $0.8(0.02,40.17)$ & $1.42(0.27,7.56)$ & $1.07(0.14,7.94)$ \\
\hline $1(0.01,127.89)$ & $1.39(0.05,40.51)$ & FPV & $1.79(0.04,88.41)$ & $1.34(0.02,75.16)$ \\
\hline $1.03(0.06,16.72)$ & $1.43(0.15,13.69)$ & $1.03(0.02,54.16)$ & CON & $0.75(0.11,5.05)$ \\
\hline $1.37(0.03,71.06)$ & $1.90(0.3,12.16)$ & $1.37(0.08,22.81)$ & $1.33(0.08,21.82)$ & ARB \\
\hline
\end{tabular}

Alleviation of Cough

Incidence of ADRs

Figure 3. Random-effects network meta-analysis of different clinical outcomes. A. network meta-analysis of nucleic acid conversion rate; B. network meta-analysis of CT improvements; $\mathrm{C}$. network meta-analysis of cough relief and ADRs incidence. Data are mean difference $(95 \% \mathrm{CI})$ of the row treatment relative to the column treatment. Bold values indicate comparisons that are statistically significant. $\mathrm{Cl}=\mathrm{credible}$ interval. REM = Remdesivir, LPV/RTV = Lopinavir/Ritonavir, ARB = Arbidol, FPV = Favipiravir, CON = Placebo or basic therapy.

ing was FPV $>$ LPV/RTV + ARB $>$ ARB $>$ CON $>$ REM $>$ LPV/RTV (Table 2).

\subsubsection{CT improvements}

There were 3 articles reporting the CT improvements upon antiviral treatments for 7 or 14 days. Closed comparison loop was formed among CON, ARB and LPV/RTV, another direct comparison was made between LPV/RTV and FPV (Fig. 2B). According to the network meta-analysis, the outcome of CT improvements 7 days after antiviral treatment were as follows: LPV/RTV+ARB [RR 2.34, 95\%CI $(1.04,5.26)]$ had better efficacy than LPV/RTV monotherapy; LPV/RTV [RR 0.57, 95\% CI $(0.35,0.93)]$ was inferior to CON. For the clinical outcome of CT improvements 14 days after antiviral treatment, LPV/RTV [RR 2.34, 95\%CI (1.04, 5.26)] was inferior to FPV, and FPV [RR 1.85, 95\%CI $(1.07,3.2)]$ was better than ARB (Fig. 3B). The SUCRA ranking (7 days CT improvements) showed that the LPV+ARB treatment achieved the highest SUCRA score, followed by CON, FPV, LPV/RTV, and ARB; Regarding the indicator of 14 days CT improvements, the ranking was $\mathrm{FPV}>\mathrm{CON}>\mathrm{LPV}>\mathrm{ARB}$ (Table 2).

\subsubsection{Improvements in clinical symptoms and treatment safety}

There were 2 articles comparing the cough alleviation and 6 articles reporting the frequencies of adverse effects. The net-work relationships of adverse effects among different interventions were shown in Fig. 2C. Closed comparison loop was formed among interventions of LPV/RTV, FPV and ARB, and other direct compar- isons were made between CON vs. LPV/RTV and CON vs. REV, respectively. However, there were no significant difference between any interventions (Fig. 3C). Though there was no difference in the random-effect model, the SUCRA score suggested that the safety ranking was FPV $>$ ARB $>$ REM $>$ CON $>$ LPV/RTV and FPV also displayed the best ranking result in alleviation of cough, followed by LPV/RTV, ARB and CON (Table 2).

\subsubsection{Efficacy in all-cause mortality}

There were 2 studies reporting all-cause mortality. Wang Yeming et al. Compared motality of REM vs. Placebo at day 7, 14 and 28 , and no significant differences were found between two interventions [16]. Cao B. et al. compared motality at day 7 and day 14 after LPV/RTV vs. Standard care initiated in severe COVID-19 [17]. 199 patients underwent randomization and the total mortality rate of COVID-19 was $6 \%$ at day 7 , with $5.1 \%$ in LPV/RTV cohort $v$ s. $7 \%$ in standard care cohort. Although the motality rate climb up to $16.1 \%$, with $15.2 \%$ in LPV/RTV cohort $v s .17 \%$ in standard care cohort, no statistic significance was found between two cohorts.

\section{Discussion}

Though the COVID-19 pandemic is currently under control in some countries, the WHO has announced COVID-19 as an international public health emergency. Thanks to the dedication of hardworking scientists and clinicians around the world, novel agents, or new activity of currently used drugs i.e. drug repurposing, such as chloroquine (CQ) and its analogue hydroxychloroquine (HCQ) 
Table 2. SUCRA Ranking of different clinical outcomes*

\begin{tabular}{|c|c|c|}
\hline Treatment & SUCRA & MeanRank \\
\hline \multicolumn{3}{|c|}{7 days nucleotide conversion rate } \\
\hline ARB & 40.9 & 4 \\
\hline $\mathrm{CON}$ & 48.3 & 3.6 \\
\hline FPV & 95.8 & 1.2 \\
\hline LPV/RTV & 2.5 & 5.9 \\
\hline LPV/RTV+ARB & 59.9 & 3 \\
\hline REM & 52.6 & 3.4 \\
\hline \multicolumn{3}{|c|}{14 days nucleotide conversion rate } \\
\hline $\mathrm{ARB}$ & 60.8 & 3 \\
\hline $\mathrm{CON}$ & 31 & 4.4 \\
\hline FPV & 83.1 & 1.8 \\
\hline LPV/RTV & 21.7 & 4.9 \\
\hline LPV/RTV+ARB & 76.5 & 2.2 \\
\hline REM & 26.8 & 4.7 \\
\hline \multicolumn{3}{|c|}{7 days $\mathrm{CT}$ improvements } \\
\hline $\mathrm{ARB}$ & 16.4 & 4.3 \\
\hline $\mathrm{CON}$ & 73.3 & 2.1 \\
\hline FPV & 54.7 & 2.8 \\
\hline LPV/RTV & 17.9 & 4.3 \\
\hline LPV/RTV+ARB & 87.7 & 1.5 \\
\hline \multicolumn{3}{|c|}{14 days CT improvements } \\
\hline $\mathrm{ARB}$ & 9 & 3.7 \\
\hline $\mathrm{CON}$ & 59.9 & 2.2 \\
\hline FPV & 96.7 & 1.1 \\
\hline LPV/RTV & 34.4 & 3 \\
\hline \multicolumn{3}{|c|}{ Incidence of ADRs } \\
\hline $\mathrm{ARB}$ & 53.7 & 2.9 \\
\hline $\mathrm{CON}$ & 47.1 & 3.1 \\
\hline FPV & 66.2 & 2.4 \\
\hline LPV/RTV & 33.4 & 3.7 \\
\hline REM & 49.6 & 3 \\
\hline \multicolumn{3}{|c|}{ Alleviation of Cough } \\
\hline $\mathrm{ARB}$ & 48.5 & 2.5 \\
\hline $\mathrm{CON}$ & 37.6 & 2.9 \\
\hline FPV & 58.3 & 2.3 \\
\hline LPV/RTV & 55.6 & 2.3 \\
\hline
\end{tabular}

*Abbreviations: $\mathrm{ARB}=$ Arbidol, $\mathrm{CON}=$ placebo or basic therapy, FPV $=$ favipiravir, $\mathrm{LPV} / \mathrm{RTV}=$ lopinavir/ritonavir, $\mathrm{REM}=$ remdesivir.

were investigated for their anti-viral activity [3]. However, the specific pharmacokinetic characteristics (long time elimination, CYP3A4 inhibition that may induce various drug-to-drug interactions, etc.) and safety concerns (e.g. cardiac side effects) of CQ and HCQ limited their clinical application [18, 19]. REM was first developed as an antiviral drug against SARS-CoV and MERSCoV [20, 21], both viruses of which suddenly disappeared in humans, thus markedly diminishing clinical research. LPV/RTV as well as ARB were proven to have activity either in in vitro studies as well as in animal models or for post-exposure prophylaxiss $[22,23]$, and they were being investigated in hundreds of registered clinical trials worldwide. According to our network meta-analysis, FPV was the best candidate antiviral drug in terms of both efficacy (i.e. CT improvements and nucleic acid positive-to-negative con- version rate) and safety.

FPV was first approved in Japan and a recent research unveiled its activity against COVID-19 [14] . FPV, as a novel RNAdependent RNA polymerase (RdRp) inhibitor, shows inhibitory effects on a wide range of RNA viruses, such as the influenza virus. Retrospective analysis of patients with Ebola virus disease (EVD) indicated that FPV treatment showed advantages in overall survival [24]. The genomic characteristics of SARS-CoV-2 is that it contains a single-stranded RNA beta-coronavirus genome harboring the RdRp gene, which shares great similarity with SARS$\mathrm{CoV}$ as well as MERS-CoV [1]. Preclinical research demonstrated the efficacy of FPV against SARS-CoV-2 in vitro (EC50 $=61.9 \mu \mathrm{M}$ determined using monkey African green kidney Vero E6 cells) [3]. Two clinical trials have investigated the comparative effectiveness and safety of FPV versus ritonavir-boosted lopinavir or ARB [14, 15], with randomized or controlled study, suggesting moderate-to-high level evidence. The most common adverse events of FPV were digestive tract reactions (nausea and diarrhea) and increased serum uric acid, side effects of which were irreversible. CFDA (China Food and Drug Administration, CFDA) approved FPV on the $16^{\text {th }}$ Feb of 2020 as an antiviral agent for COVID-19. Consequently, the results of CT improvements, nucleic acid positive-to-negative rate, the efficacy and safety of FPV were apparently encouraging. These results provide clinicians with an apparently promising candidate for COVID-19 treatment.

REM was first developed as an adenosine analogue against EBOLA virus, which undergoes incorporation into nascent viral RNA chains and results in premature termination of viral replication. Actually, it is a prodrug of an adenosine analogue that has a broad antiviral spectrum, including all human and animal coronaviruses tested in vitro. As the first antiviral drug proved to be effective against SARS-CoV-2 in vitro [3], REM got high expectations especially after the first case in the United States recovered by REM treatment [25]. There are 8 registered clinical trials in the NIH, 3 of which have been completed and the results reported. However, the results of REM seem controversial: a multicenter, randomized, double-blind clinical trial launched in severe COVID-19 patients in China indicates REM is not associated with statistically significant clinical benefits, though it shortened the time to clinical improvements [16] (NCT04257656). The clinical trial of REM in adults with mild and moderate COVID-19 was suspended due to lack of patients. NIH clinical trials named “Adaptive COVID-19 Treatment Trial" (ACTT) launched in the United States showed that REM accelerated patient recovery from advanced COVID-19. Patients who received REM had a $31 \%$ faster time to recovery than those who received placebo. Besides, a favorable mortality rate was also observed in REM group $(p=0.059)$ (NCT04280705). The ACTT trial designed to be completed on April $1^{s t}$, 2023, so we have only included Cao Bin's research in this manuscript due to lack of full data of another clinical study. In fact, the inconsistency of the REM results is related to the inconsistent criteria for efficacy evaluation. The clinical study launched by Cao Bin et al designed a six-point scale to evaluate the clinical improvements by at least two-point reduction in patients' admission status within 28 days after randomization. The primary clinical outcome of ACTT was the time to recovery within 29 days, which defined a three-point scale. Due to the circumstances of 
COVID-19 pandemic, Gilead Inc announced the latest news that the Japanese Ministry of Health, Labor and Welfare (MHLW) has approved REM as a treatment for severe patients COVID-19 patients. Generally, the activity of REM to COVID-19 patients in clinical improvements, mortality, as well as frequencies of adverse effects, needs to be confirmed in large-sample clinical study.

As for LPV/RTV, the drug-to-drug interaction and side effects could not be ignored. LPV/RTV were first designed and developed as a peptidomimetic HIV protease inhibitors and tested against COVID-19 treatment due to its previous studies on SARS-CoV $[19,26]$ and its availability at the very beginning of the epidemic in Wuhan. The clinical trial completed by Cao Bin et al focused on severe COVID-19 patients, though LPV/RTV treatment could reduce the time to clinical improvement ( 15 days versus 16 days of standard care), LPV/RTV was not associated with statistically significant clinical benefits [17]. When compared with ARB, the latter was more superior to LPV/RTV in nucleic acid positive-tonegative conversion [11]. Additionally, a clinical trial launched by Guangzhou Eighth People's Hospital demonstrated that LPV/RTV, as well as ARB had little benefit in improving the clinical outcome of mild/moderate COVID-19, and LPV/RTV was associated with adverse reactions [13] , that was a major concern of LPV/RTV in clinical application. Ritonavir-boosted lopinavir could increase the bioavailability of lopinavir through inhibition of the CYP3A enzyme [27] . Lymphopenia was the most frequent and severe adverse effect of LPV/RTV during the treatment of COVID-19 [17]. The digestive tract side effects including diarrhea, vomiting, and nausea, were more common than that observed upon FPV treatment [25] . As the CYP3A enzyme was the principal enzyme of metabolism, LPV/RTV might induce various drug-to-drug interactions [28] . Some commonly applied agents in COVID-19 treatment including CQ, HCQ as well as moxifloxacin had strong interactions with LPV/RTV, leading to increased the serum concentration of those agents. That may also result in increasing side effects of LPV/RTV. Additionally, LPV/RTV monotherapy was inferior to other interventions concerning CT improvements, nucleic acid conversion rates, and adverse reactions et al.

ARB displayed better efficacy than LPV/RTV in nucleic acid conversion rate and fewer safety concerns. ARB is a nonnucleoside analogue designed and developed by Russian scientists. As a hemagglutinin inhibitor, it could block the entry of viruses and induce the secretion of interferon and display broad antiviral activity against influenza, human respiratory syncytial (HRSV), adenovirus, Coxsackie B5, parainfluenza, Ebola (EBOV), and hepatitis B and C viruses [29]. Previous studies showed its efficacy in inhibiting SARS-CoV as well as MERSCoV [30, 31] . Based on this, professor Li Lanjuan et al., tested the antiviral activity of ARB and showed that it could significantly inhibit SARS-CoV-2 at the concentrations of $\sim 10-30 \mu \mathrm{M}$ in vitro. Due to the COVID-19 crisis, ARB was then recommended in the Chinese Clinical Guidance for the treatment COVID-19. According to our meta-analysis results, ARB was superior to LPV/RTV in the 7 days' treatment in the nucleic acid conversion rate. The incidence of adverse effects of ARB was less than LPV/RTV, though no statistical significance was found

The current literature review study has some limitations: (i) There is limited antiviral clinical research included due to lack of published data, and only three randomized clinical trials were included in our review, which may increase the risks of bias; (ii) Only one REM clinical trial was included and due to varied assessment scale, there is limited data in 28-days mortality and direct comparison. The therapeutic efficacy and safety of REM need to be validated in future studies; (iii) The majority of the sample size in most of the studies was small which may be due to the drug accessibility and the pandemic crisis.

Additionally, recent study have unveiled the fact that antioxidants and heme oxygenase contribute a lot in pathogenesis and progression of COVID-19 [32, 33, 34]. Clinical observations also reported the paradox of COVID-19 hospital admissions that fewer cigarette-smokers are admitted compared with non-smokers in general population, which could be explained by the fact that longtime smoke could induce the expression of HO-1, which plays a vital role in antioxidant and cytoprotective properties and could limit the infection's damage [32]. Ahmed S. et al. found that alveoloar type II cell SOD3 was the top-ranked gene that was most down-regulated in the elderly compared with young healthy donors through single cell RNA sequencing [33]. Taken together, the decreased ability in antioxidant may aggravate COVID-19 progression, especially in the elderly. Therapies with novel anti-oxidant agents as well as promisiong antiviral agents should be taken into consideration in order to prevent or treat COVID-19 disease.

In conclusion, FPV was superior to other antiviral agents in clinical improvement indicators (CT improvements, cough relief, nucleic acid conversion rate, and incidence of adverse reactions). ARB had advantages in nucleic acid conversion rate and the incidence of side effects. The activity of REM needs to be validated in more clinical research. Above all, the encouraging results of FPV may provide clinicians a rationale choice for COVID-19 treatment

\section{Acknowledgments}

We would like to express our gratitude to all those who dedicated themselves in the COVID-19 pandemics and thanks to all the peer reviewers and editors for their opinions and suggestions.

\section{Conflict of interest}

The authors declare no conflict of interest.

\section{Supplementary material}

Supplementary material associated with this article can be found, in the online version, at https://jmcm.imrpress.com/EN/10. 31083/j.jmcm.2020.01.003.

\section{References}

[1] Lu R, Zhao X, Li J, Niu P, Yang B, Wu H, Wang W, Song H, Huang B, Zhu N, Bi Y, Ma X, Zhan F, Wang L, Hu T, Zhou H, Hu Z, Zhou W, Zhao L, Chen J, Meng Y, Wang J, Lin Y, Yuan J, Xie Z, Ma J, Liu WJ, Wang D, Xu W, Holmes EC, Gao GF, Wu G, Chen W, Shi W and Tan W. Genomic characterisation and epidemiology of 2019 novel coronavirus: implications for virus origins and receptor binding. Lancet. 2020; 395: 565-574.

[2] The species Severe acute respiratory syndrome-related coronavirus: classifying 2019-nCoV and naming it SARS-CoV-2. Nat Microbiol. 2020; 5: 536-544

[3] Wang M, Cao R, Zhang L, Yang X, Liu J, Xu M, Shi Z, Hu Z, Zhong $\mathrm{W}$ and Xiao G. Remdesivir and chloroquine effectively inhibit the 
recently emerged novel coronavirus (2019-nCoV) in vitro. Cell Res. 2020; 30: 269-271.

[4] De Meyer S, Bojkova D, Cinatl J, Van Damme E, Buyck C, Van Loock M, Woodfall B and Ciesek S. Lack of antiviral activity of darunavir against SARS-CoV-2. Int J Infect Dis. 2020; 97: 7-10.

[5] National Health Commission of the People's Republic of China. Coronavirus Disease 2019 (COVID-19) treatment Guidelines (7th edition). www.nhc.gov.cn.

[6] National Health Commission. Chinese clinical guidance for COVID19 pneumonia diagnosis and treatment (7th edition). China National Health Commission, State Administration of Traditional Chinese Medicine. 2020. (In Chinese)

[7] Expert consensus on chloroquine phospate for the treatment of novel coronavirus pneumonia. Zhonghua Jie He He Hu Xi Za Zhi. 2020; 43(0): E019. (In Chinese)

[8] Higgins JPT, Altman DG, Gotzsche PC, Juni P, Moher D, Oxman AD, Savovic J, Schulz KF, Weeks L and Sterne JAC. The Cochrane Collaboration's tool for assessing risk of bias in randomised trials. BMJ. 2011; 343: d5928.

[9] Stang A. Critical evaluation of the Newcastle-Ottawa scale for the assessment of the quality of nonrandomized studies in meta-analyses. Eur J Epidemiol. 2010; 25: 603-605.

[10] Guyatt GH, Oxman AD, Vist GE, Kunz R, Falck-Ytter Y, AlonsoCoello P, Schünemann HJ and GRADE WG. GRADE: an emerging consensus on rating quality of evidence and strength of recommendations. BMJ. 2008; 336: 924-926.

[11] Zhu Z, Lu Z, Xu T, Chen C, Yang G, Zha T, Lu J and Xue Y. Arbidol monotherapy is superior to lopinavir/ritonavir in treating COVID-19. J Infection. 2020; 81: e21-e23.

[12] Deng L, Li C, Zeng Q, Liu X, Li X, Zhang H, Hong Z, Xia J. Arbidol comined with $\mathrm{LPV} / \mathrm{r}$ versus $\mathrm{LPV} / \mathrm{r}$ alone against coronavirus disease 2019: a retrospective cohort study. 2020; 81: e1-e5.

[13] Li Y, Xie Z, Lin W, Cai W, Wen C, Guan Y, Mo X, Wang J, Wang Y, Peng P, Chen X, Hong W, Xiao G, Liu J, Zhang L, Hu F, Li F, Zhang F, Deng X, Li L. An exploratory randomized, controlled study on the efficacy and safety of lopinavir/ritonavir or arbidol treating adult patients hospitalized with mild/moderate COVID-19(ELACOI). medRxiv. doi: https://doi.org/10.1101/2020. 03.19.20038984 [preprint].

[14] Chen C, Huang J, Cheng Z, Wu J, Chen S, Zhang Y, Chen B, Lu M, Luo Y, Zhang J, Yin P, Wang X. Favipiravir versus Arbidol for COVID-19: A Randomized Clinical Tri al.: NewsRX LLC, 2020. 389.

[15] Cai Q, Yang M, Liu D, Chen J, Shu D, Xia J, et al. Experimental treatment with favipiravir for COVID-19: An open-label control study. Engineering. 2020.

[16] Wang Y, Zhang D, Du G, Du R, Zhao J, Jin Y, Fu S, Gao L, Cheng Z, Lu Q, Hu Y, Luo G, Wang K, Lu Y, Li H, Wang S, Ruan S, Yang C, Mei C, Wang Y, Ding D, Wu F, Tang X, Ye X, Ye Y, Liu B, Yang J, Yin W, Wang A, Fan G, Zhou F, Liu Z, Gu X, Xu J, Shang L, Zhang Y, Cao L, Guo T, Wan Y, Qin H, Jiang Y, Jaki T, Hayden FG, Horby PW, Cao B and Wang C. Remdesivir in adults with severe COVID-19: a randomised, double-blind, placebo-controlled, multicentre trial. The Lancet. 2020.

[17] Cao B, Wang Y, Wen D, Liu W, Wang J, Fan G, Ruan L, Song B, Cai Y, Wei M, Li X, Xia J, et al. A trial of lopinavir-ritonavir in adults hospitalized with severe COVID-19. NEngl JMed. 2020; 382: 17871799.

[18] Yao X, Ye F, Zhang M, Cui C, Huang B, Niu P, Liu X, Zhao L, Dong E, Song C, Zhan S, Lu R, Li H, Tan W and Liu D. In vitro antiviral activity and projection of optimized dosing design of hydroxychloroquine for the treatment of severe acute respiratory syndrome coronavirus 2 (SARS-CoV-2). Clin Infect Dis. 2020.

[19] Chen F, Chan KH, Jiang Y, Kao RY, Lu HT, Fan KW, Cheng VC, Tsui WH, Hung IF, Lee TS, Guan Y, Peiris JS and Yuen KY. In vitro susceptibility of 10 clinical isolates of SARS coronavirus to selected antiviral compounds. J Clin Virol. 2004; 31: 69-75.

[20] Chatre C, Roubille F, Vernhet H, Jorgensen C and Pers Y. Cardiac Complications attributed to chloroquine and hydroxychloroquine: a systematic review of the literature. Drug Safety. 2018; 41: 919-931.

[21] Marmor MF, Kellner U, Lai TYY, Melles RB and Mieler WF. Recommendations on Screening for Chloroquine and Hydroxychloroquine Retinopathy (2016 Revision). Ophthalmology. 2016; 123: 1386-1394.

[22] Sheahan TP, Sims AC, Leist SR, Schafer A, Won J, Brown AJ, Montgomery SA, Hogg A, Babusis D, Clarke MO, Spahn JE, Bauer L, Sellers S, Porter D, Feng JY, Cihlar T, Jordan R, Denison MR and Baric RS. Comparative therapeutic efficacy of remdesivir and combination lopinavir, ritonavir, and interferon beta against MERS-CoV. Nat Commun. 2020; 11: 222.

[23] Potential of arbidol for post-exposure prophylaxis of COVID-19 transmission-preliminary report of a retrospective case-control study. 2020; chinaXiv: 202002.00065v1.

[24] Bai CQ, Mu JS, Kargbo D, Song YB, Niu WK, Nie WM, Kanu A, Liu WW, Wang YP, Dafae F, Yan T, Hu Y, Deng YQ, Lu HJ, Yang F, Zhang XG, Sun Y, Cao YX, Su HX, Sun Y, Liu WS, Wang CY, Qian J, Liu L, Wang H, Tong YG, Liu ZY, Chen YS, Wang HQ, Kargbo B, Gao GF and Jiang JF. Clinical and Virological Characteristics of Ebola Virus Disease Patients Treated With Favipiravir (T-705)-Sierra Leone, 2014. Clin Infect Dis. 2016; 63: 1288-1294.

[25] Holshue ML, DeBolt C, Lindquist S, Lofy KH, Wiesman J, Bruce H, Spitters C, Ericson K, Wilkerson S, Tural A, Diaz G, Cohn A, Fox L, Patel A, Gerber SI, Kim L, Tong S, Lu X, Lindstrom S, Pallansch MA, Weldon WC, Biggs HM, Uyeki TM and Pillai SK. First Case of 2019 Novel Coronavirus in the United States. N Engl J Med. 2020; 382: 929-936.

[26] Chu CM, Cheng VC, Hung IF, Wong MM, Chan KH, Chan KS, Kao RY, Poon LL, Wong CL, Guan Y, Peiris JS and Yuen KY. Role of lopinavir/ritonavir in the treatment of SARS: initial virological and clinical findings. Thorax. 2004; 59: 252-256.

[27] van Waterschoot RA, ter Heine R, Wagenaar E, van der Kruijssen CM, Rooswinkel RW, Huitema AD, Beijnen JH and Schinkel AH. Effects of cytochrome P450 3A (CYP3A) and the drug transporters P-glycoprotein (MDR1/ABCB1) and MRP2 (ABCC2) on the pharmacokinetics of lopinavir. Br J Pharmacol. 2010; 160: 1224-1233.

[28] Genetic Profiles in Pharmacogenes Indicate Personalized Drug Therapy for COVID-19 Running title: COVID-19 pharmacogenetics.

[29] Kadam RU and Wilson IA. Structural basis of influenza virus fusion inhibition by the antiviral drug Arbidol. Proc Natl Acad Sci USA. 2017; 114: 206-214.

[30] Khamitov RA, Loginova SI, Shchukina VN, Borisevich SV, Maksimov VA and Shuster AM. Antiviral activity of arbidol and its derivatives against the pathogen of severe acute respiratory syndrome in the cell cultures. Voprosy virusologii. 2008; 53: 9.

[31] Ji Xiaoguang, Zhao Yanhong, Zhang Min, Zhao Jinghua, Wang Jingyan, The experimental study of the anti-SARS-CoV effect of arbidole.Pharm J Chin PLA, 2004; 04: p274-276.

[32] Hooper PL. COVID-19 and heme oxygenase: novel insight into the disease and potential therapies. Cell Stress and Chaperones. 2020.

[33] Abouhashem AS, Singh K, Azzazy HME and Sen CK. Is low alveolar type II CellSOD3 in the lungs of elderly linked to the observed severity of COVID-19? Antioxid Redox Sign. 2020; 33: 59-65.

[34] Livan D, Fernando M. Oxidative Stress as Key Player in Severe Acute Respiratory Syndrome Coronavirus (SARS-CoV) infection.Archives of Medical Research. 2020; 51(5): 384. 